\title{
Operations on partially ordered sets and rational identities of type $A$
}

\author{
Adrien Boussicault \\ Institut Gaspard Monge, Université Paris-Est, Marne-la-Vallé, France \\ received $13^{\text {th }}$ February 2009, revised $1^{\text {st }}$ April 2013, accepted $2^{\text {nd }}$ April 2013.
}

We consider the family of rational functions $\psi_{w}=\prod\left(x_{w_{i}}-x_{w_{i+1}}\right)^{-1}$ indexed by words with no repetition. We study the combinatorics of the sums $\Psi_{P}$ of the functions $\psi_{w}$ when $w$ describes the linear extensions of a given poset $P$. In particular, we point out the connexions between some transformations on posets and elementary operations on the fraction $\Psi_{P}$. We prove that the denominator of $\Psi_{P}$ has a closed expression in terms of the Hasse diagram of $P$, and we compute its numerator in some special cases. We show that the computation of $\Psi_{P}$ can be reduced to the case of bipartite posets. Finally, we compute the numerators associated to some special bipartite graphs as Schubert polynomials.

Keywords: poset, rational function, Schubert polynomials

\section{Introduction}

For each word $w$ without repetition, we define the rational function

$$
\psi_{w}:=\frac{1}{\left(x_{w_{1}}-x_{w_{2}}\right) \cdot\left(x_{w_{2}}-x_{w_{3}}\right) \cdots\left(x_{w_{n-1}}-x_{w_{n}}\right)}
$$

where $w_{1} \ldots w_{n}$ are the letters of $w$. Permutations are special words with no repetition, and summing these functions on intervals of the permutohedron (i.e., for the weak order) gives remarkable properties. For example, we will show that, when a permutation $\sigma$ avoids some patterns, the sum over the initial interval $[I d, \sigma]$ can be simplified to a product $\prod\left(x_{i}-x_{j}\right)^{-1}$ where the product runs over the edges $(i, j)$ of a particular tree.

These patterns are known to characterise some families of Schubert varieties. Schubert varieties are indexed by permutations, and the varieties which are non-singular are those whose indexing permutation does not contain the pattern 2143 nor 1324. In (2), Cortez has described geometrical properties of Schubert varieties for permutations avoiding 1324 and 2143 (i)] This was further clarified by Woo and Yong in (6),

(i) A permutation $\sigma$ avoids the pattern 1324 if there exist no integers $1 \leq i_{1}<i_{2}<i_{3}<i_{4} \leq n$ satisfying $\sigma_{i_{1}}<\sigma_{i_{3}}<\sigma_{i_{2}}<$ $\sigma_{i_{4}}$. A permutation $\sigma$ contains the pattern $\overline{2143}$ if for some indices $1 \leq i_{1}<i_{2}<i_{3}<i_{4} \leq n$ we have $\sigma_{i_{2}} \leq \sigma_{i_{1}} \leq \sigma_{i_{4}} \leq$ $\sigma_{i_{3}}$ with the further restriction that there is no $i_{1} \leq j \leq i_{4}$ such that $\sigma_{i_{1}} \leq \sigma_{j} \leq \sigma_{i_{4}}$. 
and Butler and Bousquet-Mélou in (1). They used the fact that an initial interval $[I d, \sigma]$ is the set of linear extensions of a partially ordered set whose Hasse diagram is a tree when $\sigma$ avoids 1324 and $\overline{2143}$.

Surprisingly, the same patterns occur in the study of the sum

$$
\Psi_{P}:=\sum_{w \in \mathcal{L}(P)} \psi_{w}
$$

where $\mathcal{L}(P)$ is the set of linear extensions of $P$. In fact, our work is closely connected to a study of Greene (3) on rational identities related to the Murnaghan-Nakayama formula for $S_{n}$ (type $A$ ). Greene gave in (3) a closed expression for the $\Psi_{P}$ of a planar poset $P$. He showed the equality

$$
\Psi_{P}=\left\{\begin{array}{cl}
0 & \text { if } \mathrm{P} \text { is a disconnected graph, } \\
\prod_{a, b \in P}\left(x_{a}-x_{b}\right)^{\mu_{P}(a, b)} & \text { if } \mathrm{P} \text { is a connected graph, }
\end{array}\right.
$$

where $\mu(x, y)$ is the Möbius function of the poset $P$. In the case of a permutation avoiding the patterns 1324 and $\overline{2143}$, the poset is planar, and the Möbius function takes only values 0 or -1 . Therefore (Corollary 4.1) the numerator of $\Psi_{P}$ equals 1 if and only if the Hasse diagram of $P$ is acyclic.

The aim of this paper consists in pointing out the connexions between some operations on posets and rational identities involving the $\Psi_{P}$. To study the rational functions $\Psi_{P}$, we introduce some operations on posets in Section 2, and describe in Section 3 the identities on the rational functions that these operations induce. Finally, we give some explicit examples in Section 4. acyclic posets, 1-cycle posets and $\lambda$ complete posets (related to Schubert polynomials ).

\section{Operations on partially ordered sets}

We will see that the knowledge of the Hasse diagram of $P$ gives some properties of the function $\Psi_{P}$. In this section we study four operations on posets (considered as graphs): collapse, operation of sorting a subset, contraction and suppression of extremal elements.

\subsection{Basic definitions}

We recall that a partially ordered set (poset) $P=(A, \leq)$ is a set $A$ endowed with a reflexive, antisymmetric and transitive binary relation. For simplicity, when there is no ambiguity, we will use $a \in P$ instead of $a \in A$.

A total order $T$ is a linear extension of a poset $P$ if, whenever $a \leq b$ in $P$ there holds $a \leq b$ in $T$. We denote by $\mathcal{L}(P)$ the set of linear extensions of $P$.

Classically, the covering relation is the minima $\left.{ }^{(\text {iii }}\right)$ relation whose transitive closure is $\leq$. This relation will be denoted by $\preceq$. The Hasse diagram of $P$, denoted by $H(P)$, is the oriented graph of the covering relation of $P$, drawn in such a way that if $a \leq b$, then $b$ is drawn to the right of $a$ (iii). The Hasse diagram displays the minimal set of relations generating $P$ by transitivity.

The set of the inner vertices of $P$, that is, those being neither minimal nor maximal for $\leq$, will be denoted by $\operatorname{In}(P)$. Its complementary set is called the boundary of $P: \operatorname{Bound}(P):=P \backslash \operatorname{In}(P)$

(ii) as a subset of $P \times P$.

(iii) Usually, Hasse diagrams are drawn from bottom to top, but this representation takes more space and is less natural for our purposes. 


\subsection{Permutations and posets}

Consider the symmetric group $\mathfrak{S}_{n}$ endowed with the weak order.

The weak order (also called right permutohedron order) is the order on permutations obtained by defining the successors of a permutation $\sigma$ as the permutations $\sigma . s_{i}$ if this permutation has more inversions than $\sigma$, where $s_{i}=(i, i+1)$ exchanges the numbers at places $i$ and $i+1$ of $\sigma$.

For any pair $(\sigma, \tau)$ of permutations, one constructs the interval $[\sigma, \tau]$ as the set of permutations greater than or equal to $\sigma$ and lower than or equal to $\tau$.

For example, the interval $[123456,132564]$ in $\mathfrak{S}_{6}$ contains exactly the permutations

$$
[123456,132564]=\{132564,123564,132546,123546,132456,123456\} .
$$

Proposition 2.1 Let $\sigma$ be a permutation. There exists a poset $\mathbb{P}_{\sigma}$ such that $\mathcal{L}\left(\mathbb{P}_{\sigma}\right)=[I d, \sigma]$. Moroever, the Hasse diagram of $\mathbb{P}_{\sigma}$ is obtained applying Algorithm 1$]$

Proof: Let $\leq_{\sigma}$ be the binary relation on $\{1, \ldots, n\}$ defined by:

$$
i \leq_{\sigma} j \quad \text { if and only if } \quad i \leq j \text { and } \sigma_{i} \leq \sigma_{j} .
$$

The binary relation $\leq_{\sigma}$ is a partial order on $\{1, \ldots, n\}$. Indeed, reflexivity, transitivity and antisymmetry of $>_{\sigma}$ are deduced from reflexivity, transitivity and antisymmetry of $\leq$.

Let $\mathbb{P}_{\sigma}$ be the poset defined by:

$$
\mathbb{P}_{\sigma}=\left(\{1, \ldots, n\}, \leq_{\sigma}\right) .
$$

The interval $[I d, \sigma]$ is the set of permutations obtained from $\sigma$ by permuting recursively all consecutive decreasing pairs of letters of $\sigma$. It is well known that $[i d, \sigma]$ is exactly the set

$$
[I d, \sigma]=\left\{\tau \in \mathfrak{S}_{n} \mid \text { if } i \leq j \text { and } \sigma_{i}^{-1} \leq \sigma_{j}^{-1} \text { then } \tau_{i}^{-1} \leq \tau_{j}^{-1}\right\}
$$

which is, by definition, the set of linear extensions of $\mathbb{P}_{\sigma}$.

The Hasse diagram of such a poset is built by using Algorithm 1. Indeed, the first ten steps draw each vertices of the graph satisfying that if $i<j$ and $\sigma_{i}<\sigma_{j}$ then $\sigma_{i}$ is draw to the right of $\sigma_{j}$. Step 11 draws the edges of the Hasse diagram.

Example 2.2 Figure 1 shows an execution of Algorithm 1$]$

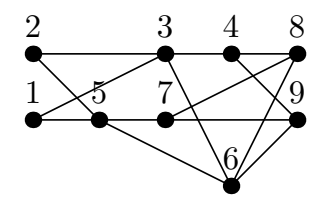

Fig. 1: Hasse diagram of $\mathbb{P}_{215736498}$ 


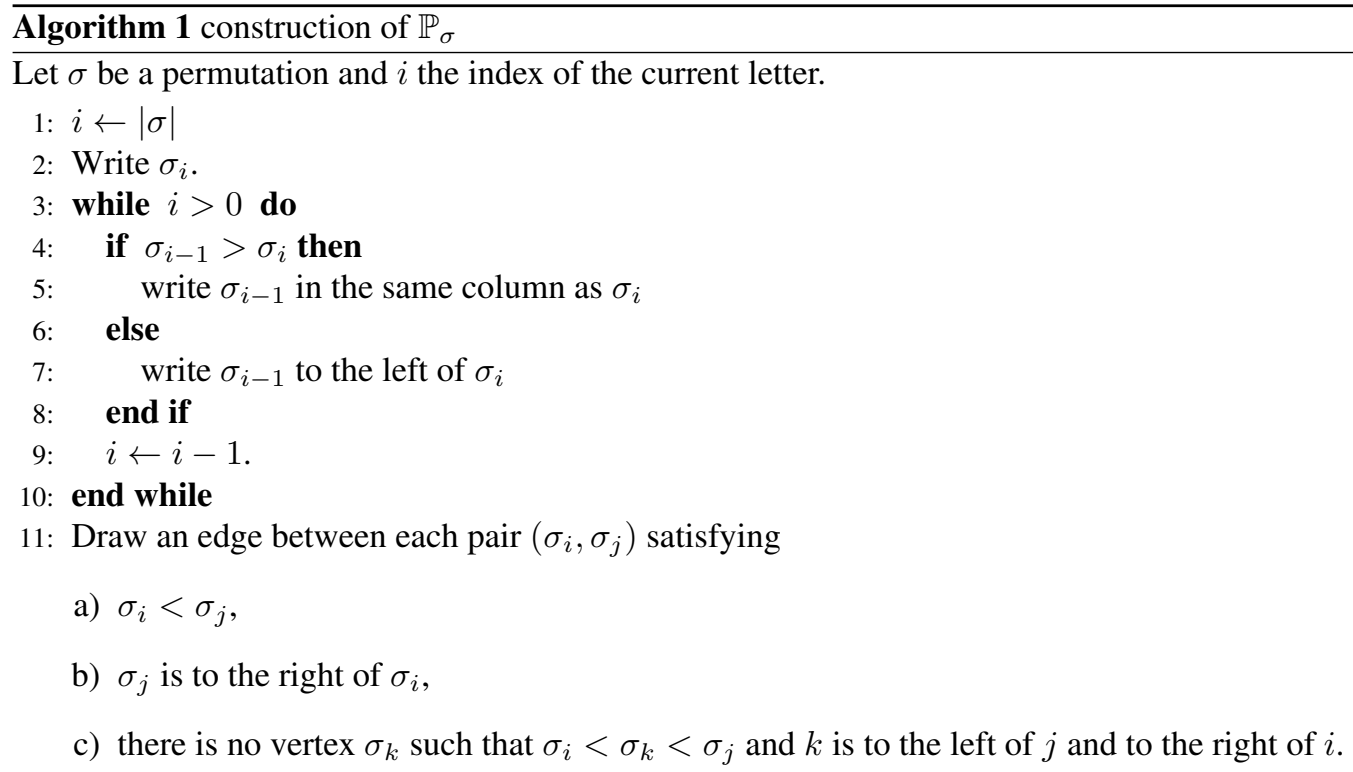

12: The graph obtained is the Hasse diagram of the poset $\mathbb{P}_{\sigma}$.

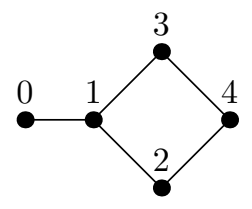

Fig. 2: 0 is a free vertex and $(0,1)$ is a non-inner edge

\subsection{Collapses}

Hasse diagrams can be viewed as non-oriented graphs. We will use notations and definitions given in (9). In his book (9), Giblin defined a free vertex as a vertex belonging to exactly one edge. An edge with no free vertex is an inner edge.

Example 2.3 The poset $P$ (see Figure 2) has only one free edge: 0 . The only non-inner edge is $(0,1)$.

The action which consists in removing a non-inner edge together with one of its free vertices is an elementary collapse.

A collapse is a sequence of elementary collapses. We will denote by $\operatorname{Coll}(\mathcal{G})$ the collapsed graph $\mathcal{G}$, that is the unique (up to relabeling) maximal subgraph of $\mathcal{G}$ without free vertices.

Example 2.4 Figure 3 shows the collapsed graph of Figure 2 




Fig. 3: A collapse

\subsection{Subposets and linear extensions}

Let $P=(A, \leq)$ be a poset. Each subset $A^{\prime} \subset A$ can be naturally endowed with the partial order induced by $P$ which is the projection on $A^{\prime}$ of the order of $P$. Let us denote such a poset by $P^{\prime}$. Classically, we will say that $P^{\prime}$ is a subposet of $P$ whose support is $A^{\prime}$. If $w$ is a linear extension of $P^{\prime}$, the transitive relation generated by $\leq$ and $\leq_{w}$ is a partial order on $A$ and the corresponding poset will be denoted by $P_{w}$.

Lemma 2.5 Let $P=(A, \leq)$ be a poset, $A^{\prime} \subset A$ and $P^{\prime}=\left(A^{\prime}, \leq\right)$, we have

$$
\mathcal{L}(P)=\bigsqcup_{w \in \mathcal{L}\left(P^{\prime}\right)} \mathcal{L}\left(P_{w}\right)
$$

where $\bigsqcup$ denotes the disjoint union.

Proof: Let us prove first that $\mathcal{L}(P)=\bigcup_{w \in \mathcal{L}\left(P^{\prime}\right)} \mathcal{L}\left(P_{w}\right)$. By definition of $P_{w}$ one has the inclusion $\bigcup_{w \in \mathcal{L}\left(P^{\prime}\right)} \mathcal{L}\left(P_{w}\right) \subset \mathcal{L}(P)$. Conversely, each linear extension of $P$ induces a total order on $P^{\prime}$. Hence, $\mathcal{L}(P)=\bigcup_{w \in \mathcal{L}\left(P^{\prime}\right)} \mathcal{L}\left(P_{w}\right)$.

Furthermore, if $w$ and $w^{\prime}$ are two distinct extensions of $P^{\prime}$ then, obviously, $\mathcal{L}\left(P_{w}\right) \cap \mathcal{L}\left(P_{w^{\prime}}\right)=\emptyset$.

Example 2.6 The linear extensions of the poset $P$ (see Figure 4) can be partitioned in disjoint subsets indexed by the linear extensions of the subposet $P^{\prime}$.

$$
\begin{gathered}
\mathcal{L}(P)=\mathcal{L}\left(P_{456}\right) \sqcup \mathcal{L}\left(P_{465}\right) \\
\mathcal{L}\left(P_{456}\right)=\{123456,213456,124356,214356\} \\
\mathcal{L}\left(P_{465}\right)=\{123465,213465,124365,214365\} \\
P=P_{5}^{\prime}=?
\end{gathered}
$$

Fig. 4: A poset $P$ with a subposet $P^{\prime}$. 


\subsection{Contractions}

We describe in Proposition 2.10 an operation on posets called contraction. This operation is fundamental since it allows to simplify posets by decreasing the number of edges. Before defining contraction, we need to consider the Lemmas 2.7, 2.8 and 2.9.

We denote the negation of a binary relation $\leq$ by $\not$.

Lemma 2.7 Let $\leq_{1}$ and $\leq_{2}$ be two antisymmetric relations on $A$ such that $a \leq_{2} b$ implies $b \leq_{1} a$ or $b=a$. The binary relation $a \leq_{3} b$ defined by $a \leq_{1} b$ or $a \leq_{2} b$ is antisymmetric.

Proof: Let $a, b$ be two elements of $A$ such that $a \leq_{3} b$ and $b \leq_{3} a$. One needs to examine four cases.

i) if $a \leq_{1} b$ and $b \leq_{1} a$ then $a=b$ because $\leq_{1}$ is antisymmetric.

ii) if $a \leq_{1} b$ and $b \leq_{2} a$ then, by hypothesis, $a \leq_{1} b$ and $\left(a \leq_{1} b\right.$ or $\left.b=a\right)$ which implies $b=a$.

iii) The case $a \leq_{2} b$ and $b \leq_{1} a$ is similar with the previous statement.

iv) if $a \leq_{2} b$ and $b \leq_{2} a$ than $a=b$ because $\leq_{2}$ is antisymmetric.

Let $P=(A, \leq)$ be a poset and $c, d$ two elements of $A$ satisfying $c \preceq d$. We will denote by $a \leq_{2} b$ the relation defined by $((a \leq c)$ or $(a \leq d))$ and $((c \leq b)$ or $(d \leq b))$.

Lemma 2.8 The relation $a \leq_{2} b$ is antisymmetric on $A \backslash\{d\}$.

Proof: By definition, $a \leq_{2} b$ and $b \leq_{2} a$ is equivalent to

$$
(a \leq c \text { or } a \leq d) \text { and }(c \leq a \text { or } d \leq a) \text { and }(b \leq c \text { or } b \leq d) \text { and }(c \leq b \text { or } d \leq b) .
$$

Since $c \preceq d$, we deduce that $(a=c$ or $a=d)$ and $(b=c$ or $b=d)$. Remarking that $a \neq d$ and $b \neq d$, we obtain $a=b=c$.

Lemma 2.9 Let $a, b$ be two elements of $A \backslash\{d\}$. Then $a \leq_{2} b$ implies $(b \not \leq a$ or $a=b)$.

Proof: Let $a, b$ be two elements of $A \backslash\{d\}$ satisfying $a \leq_{2} b$. By definition of $\leq_{2}$ we have $((a \leq c)$ or $(a \leq$ $d))$ and $((c \leq b)$ or $(d \leq b))$. Since $c \preceq d$, the previous formula implies $a \leq b$ or $(a \leq d$ and $c \leq b)$. If $b \leq a$, we deduce that $c \leq b \leq a \leq d$. Since $c \preceq d, a \neq d$ and $b \neq d$, we have $a=b=c$ and we obtain

$$
a \leq_{2} b \Rightarrow(a \leq b \text { or } a, b \text { are not comparable }) \Rightarrow(b \not \leq a \text { or } a=b) .
$$

We can now define the contraction.

Proposition 2.10 Let $P=(A, \leq)$ be a poset and $c, d$ be two elements of $A$. If $c \preceq d$, then the relation $\leq_{d=c}$ defined on $A \backslash\{d\}$ by

$$
a \leq_{d=c} b \Leftrightarrow(a \leq b) \text { or }((a \leq c) \text { or }(a \leq d)) \text { and }((c \leq b) \text { or }(d \leq b))
$$

is a partial order. 
Proof: The reflexivity and transitivity of $\leq_{d=c}$ follow straightforwardly from the reflexivity and transitivity of $\leq$.

By Lemmas 2.7 and 2.8, the relation $\leq_{2}$ is antisymmetric, and $a \leq_{2} b$ implies $b \not \leq a$ or $a=b$. We deduce (cf. Lemma 2.9) that $\leq_{d=c}$ is antisymmetric.

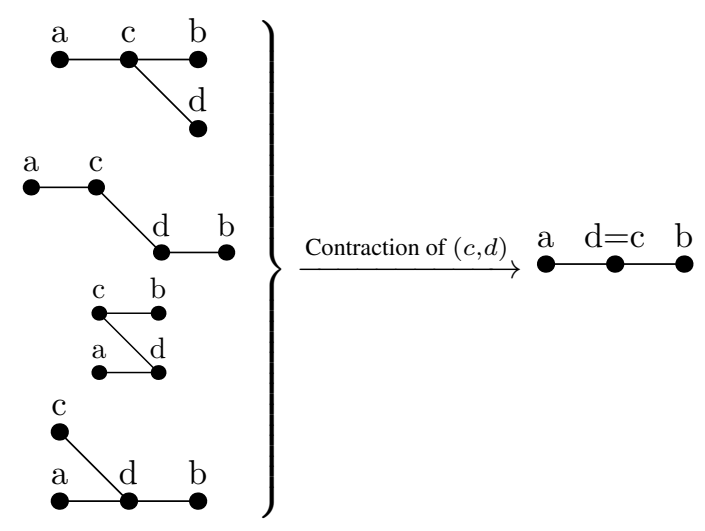

Fig. 5: Contraction of the covering relation $(c, d)$.

When $c$ covers $d$, we denote by $P_{d=c}=\left(A \backslash\{d\}, \leq_{d=c}\right)$ the contraction of the edge $(c, d)$.

Proposition 2.11 Let $P$ be a poset and $(c, d)$ be an edge in $H(P)$. Then $w^{\prime} c d w^{\prime \prime}$ is a linear extension of $P$ if and only if $w^{\prime} c w^{\prime \prime}$ is a linear extension of $P_{d=c}$.

Proof: We will denote by $i_{z}$ the position of $z$ in the world $w^{\prime} c d w^{\prime \prime}$ and by $j_{z}$ the position of $z$ in the word $w^{\prime} c w^{\prime \prime}$.

Suppose that $w^{\prime} c d w^{\prime \prime}$ is a linear extension of $P=(A, \leq)$. Let $a, b$ be two elements of $\mathbb{A} \backslash\{d\}$ such that $a \leq_{P_{d=c}} b$ and $a \neq b$. By definition of $\leq_{P_{d=c}}$, we have $(a \leq b)$ or $((a \leq c$ or $a \leq d)$ and $(c \leq b$ or $d \leq$ $b)$ ). Since $w^{\prime} c d w^{\prime \prime}$ is a linear extension, $(a \leq b)$ implies $i_{a} \leq i_{b}$. But $i_{d}=i_{c}+1, a \neq d$ and $c \neq d$, hence $((a \leq c$ or $a \leq d)$ and $(c \leq b$ or $d \leq b))$ implies $i_{a} \leq i_{c} \leq i_{b}$. Finally, in all the cases, if $a \leq_{P_{d=c}} b$ then $i_{a} \leq i_{b}$ and equivalently $w^{\prime} c w^{\prime \prime} \in \mathcal{L}\left(P_{d=c}\right)$.

Conversely, suppose that $w^{\prime} c w^{\prime \prime}$ is a linear extension of $P_{d=c}$. Let $a, b$ be two elements of $A$ such that $a \leq b$ and $a \neq b$. If $a \neq d$ and $b \neq d$ then $a \leq b$ implies $a \leq_{P_{d=c}} b$ and $j_{a} \leq j_{b}$. If $a=d$ or $b=d$ then $a \leq d$ and $d \leq b$ which implies $a \leq_{P_{d=c}} b$ and $j_{a} \leq j_{b}$. Then in all the cases, $a \leq b$ implies $j_{a} \leq j_{b}$. Equivalently, $w^{\prime} c d w^{\prime \prime}$ is a linear extension of $P$.

Example 2.12 The edge $(4,5)$ of the poset $P$ (see Figure 6) can be contracted. The linear extensions of $P_{5=4}$ are

$$
\mathcal{L}\left(P_{5=4}\right)=\{1234,2134\},
$$

and, the set obtained by removing all words of $\mathcal{L}(P)$ with no factor 45 has the same size than $\mathcal{L}\left(P_{5=4}\right)$.

$$
\left\{w^{\prime} 45 w^{\prime \prime} \in \mathcal{L}(P)\right\}=\{12345,21345\}
$$




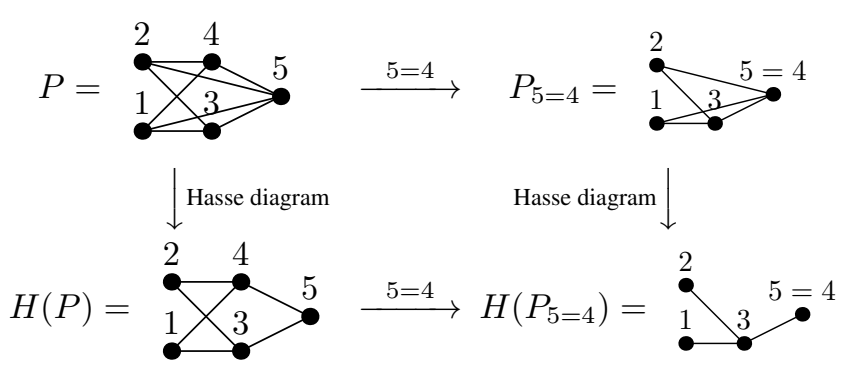

Fig. 6: The poset $P$ and $P_{5=4}$, their Hasse diagrams and their linear extensions.

\subsection{Decontraction}

In the previous section, we have described the operation of contraction. We will now show that all posets can be obtained from bipartite posets. A poset is bipartite if it has only extremal elements.

Theorem 2.13 Each poset can be obtained from a bipartite poset by applying a succession of contractions.

Proof: Consider a poset $P$. We construct a new poset $\bar{P}$ by duplicating each vertex of $\operatorname{In}(P)$

$$
\bar{P}=P \cup\{\bar{a} \mid a \in \operatorname{In}(P)\} .
$$

The poset $\bar{P}$ is endowed with the relation $\leq^{\prime}$ defined by $b \leq^{\prime} c$ if and only if one of the following statements is true:

1. $b=c$,

2. $c=\bar{a}$ with $a \in \operatorname{In}(P), b \in P$ and $b \preceq a$,

3. $b \in P, c \in \operatorname{Bound}(P)$ and $b \preceq c$,

4. $c=\bar{b}$ with $b \in \operatorname{In}(P)$.

The relation $\leq '$ is reflexive because of the first rule of construction. By construction, each element of $\bar{P}$ is either minimal or maximal. We obtain that $\leq^{\prime}$ is transitive and antisymmetric. Finally, when contracting the edges $\{(a, \bar{a}) \mid a \in \operatorname{In}(P)\}$, the poset $P$ is recovered.

Let $P$ be a poset. We will call the poset $\bar{P}$ obtained in the proof of Theorem 2.13 the decontraction of $P$.

Example 2.14 The posets $P^{\prime}$ (see Figure 7) are obtained by decontraction of the poset $P$.

\subsection{Suppression of extremal elements}

Another way to simplify posets consists in removing extremal vertices. Proposition 2.15 shows that linear extensions beginning with an extremal element $c$ are equivalent to linear extensions of a new poset obtained by removing $c$ from $P$. We will see in Subsection 3.2 that this property admits an interpretation in terms of rational functions. 


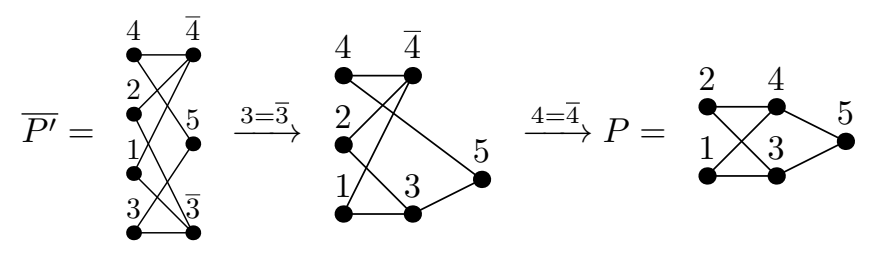

Fig. 7: The poset $P$ is the contraction of the bipartite poset $\overline{P^{\prime}}$.

Proposition 2.15 Assume that $c$ is a minimal (resp. maximal) vertex. Then, $c w$ (resp. wc) is a linear extension of $P$ if and only if $w$ is a linear extension of $P \backslash\{c\}$.

Proof: Suppose that $c w$ is a linear extension of $P$ and let $a, b$ be two elements of $P \backslash\{c\}$. Hence, if $a \leq_{P \backslash\{c\}} b$ then $a \leq_{P} b$ which implies $a \leq_{w} b$ and $w \in \mathcal{L}(P \backslash(\{c\}))$.

Conversely, suppose that $w$ is a linear extension of $P \backslash\{c\}$. Let $a, b \in P$ be such that $a \leq_{P} b$. If $a \neq c$ then, since $c$ is minimal, we have $b \neq c$. This implies $a \leq_{P \backslash\{c\}} b$ and $a \leq_{w} b$. So we have $a \leq_{c w} b$. If $a=c$, then trivially $a \leq_{c w} b$. We deduce that $c w \in \mathcal{L}(P)$.

Example 2.16 The vertex 4 of the poset $P$ (see Figure 8) can be deleted. The linear extensions of $P \backslash 4$ are

$$
\mathcal{L}(P \backslash 4)=\{123,213\}
$$

and, the set obtained by setting all word of $\mathcal{L}(P)$ having 4 in the last position has the same size than $\mathcal{L}(P \backslash 4)$.

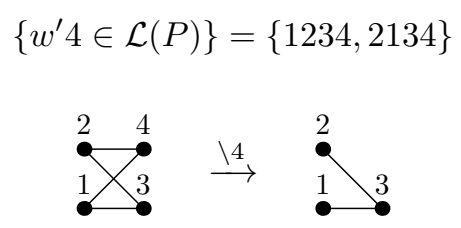

Fig. 8: Suppression of the extremal element 4.

\section{Operations on rational functions}

In the previous section we have described some operations on posets. We will now see the connexions with the rational functions $\Psi_{P}$.

\subsection{Residues and contractions}

In this subsection, we show that the fraction $\Psi_{P_{c=d}}$ can be obtained from $\Psi_{P}$ by applying a residue.

Theorem 3.1 Let $c$ and $d$ be two elements of a poset $P$. We have

$$
\lim _{x_{d} \rightarrow x_{c}}\left(x_{c}-x_{d}\right) \cdot \Psi_{P}= \begin{cases}\Psi_{P_{d=c}} & \text { if }(c, d) \text { is an edge of the Hasse diagram of } P, \\ 0 & \text { otherwise. }\end{cases}
$$


Proof: Taking the residue at $x_{c}=x_{d}$ in $\Psi_{P}$, we get

$$
\lim _{x_{d} \rightarrow x_{c}}\left(x_{c}-x_{d}\right) \Psi_{P}=\lim _{x_{d} \rightarrow x_{c}} \sum_{w \in \mathcal{L}(P)}\left(x_{c}-x_{d}\right) \psi_{w} .
$$

If neither $c d$ nor $d c$ are factors of $w$, then $\left(x_{c}-x_{d}\right)$ is a factor neither of $\operatorname{Den}\left(\psi_{w}\right)$ nor $N u m\left(\psi_{w}\right)$. It follows that $\lim _{x_{d} \rightarrow x_{c}}\left(x_{c}-x_{d}\right) \psi_{w}=0$.

If either $c d$ or $d c$ are factors of $w$, then $\left(x_{c}-x_{d}\right)$ is a factor of $\operatorname{Den}\left(\psi_{w}\right)$ with multiplicity 1 . It follows that the $\lim _{x_{d} \rightarrow x_{c}}\left(x_{c}-x_{d}\right) \psi_{w}$ converges.

We conclude that sum and limit can be permuted in Equation (1) to obtain

$$
\lim _{x_{d} \rightarrow x_{c}}\left(x_{c}-x_{d}\right) \Psi_{P}=\sum \lim _{x_{d} \rightarrow x_{c}}\left(x_{c}-x_{d}\right) \psi_{w},
$$

where the sum runs over the linear extensions $w$ of $P$ having $c d$ and $d c$ as factor (when considered as a word).

We need to consider three cases:

1) If $c$ and $d$ are not comparable. Obviously, the word $w^{\prime} c d w^{\prime \prime}$ is a linear extension of $P$ if and only if $w^{\prime} d c w^{\prime \prime}$ is also a linear extension of $P$. Hence, by considering the pairs $\psi_{w^{\prime} c d w^{\prime \prime}}$ and $\psi_{w^{\prime} d c w^{\prime \prime}}$ in [2], we obtain

$$
\lim _{x_{d} \rightarrow x_{c}}\left(x_{c}-x_{d}\right) \Psi_{P}=\sum_{w^{\prime} c d w^{\prime \prime} \in \mathcal{L}(P)} \lim _{x_{d} \rightarrow x_{c}}\left(x_{c}-x_{d}\right)\left[\psi_{w^{\prime} c d w^{\prime \prime}}+\psi_{w^{\prime} d c w^{\prime \prime}}\right] .
$$

Setting $f\left(x_{c}, x_{d}\right)=\left(x_{c}-x_{d}\right) \psi_{w^{\prime} c d w^{\prime \prime}}$, one has $f\left(x_{d}, x_{c}\right)=-\left(x_{c}-x_{d}\right) \psi_{w^{\prime} d c w^{\prime \prime}}$. Hence,

$$
\lim _{x_{d} \rightarrow x_{c}}\left(x_{c}-x_{d}\right)\left[\psi_{w^{\prime} c d w^{\prime \prime}}+\psi_{w^{\prime} d c w^{\prime \prime}}\right]=\lim _{x_{d} \rightarrow x_{c}}\left(f\left(x_{c}, x_{d}\right)-f\left(x_{d}, x_{c}\right)\right)=0 .
$$

Finally, by using Equations $(3)$ and 44 , we deduce that $\lim _{x_{c} \rightarrow x_{d}}\left(x_{c}-x_{d}\right) \Psi_{P}=0$.

2) If $c$ and $d$ are comparable but $c \npreceq d$ and $d \npreceq c$. Assuming that $c \leq d$ (the other case being similar), there is at least one element $a$ such that $c \leq a \leq d$. Then $\mathcal{L}(P)$ contains no word having neither $c d$ nor $d c$ as a factor and the residue $\lim _{x_{d} \rightarrow x_{c}}\left(x_{c}-x_{d}\right) \Psi_{P}$ equals 0 .

3) If $c \preceq d$ (the case $d \preceq c$ is similar), one has

$$
\lim _{x_{d} \rightarrow x_{c}}\left(x_{c}-x_{d}\right) \Psi_{P}=\sum \lim _{x_{d} \rightarrow x_{c}}\left(x_{c}-x_{d}\right) \Psi_{w},
$$

where the sum runs over the linear extensions $w$ of $P$ satisfying $w=w^{\prime} c d w^{\prime \prime}$. From Proposition 2.11. one has

$$
\lim _{x_{d} \rightarrow x_{c}}\left(x_{c}-x_{d}\right) \Psi_{P}=\sum_{w^{\prime} c w^{\prime \prime} \in \mathcal{L}\left(P_{d=c}\right)} \lim _{x_{d} \rightarrow x_{c}}\left(x_{c}-x_{d}\right) \Psi_{w^{\prime} c d w^{\prime \prime}} .
$$

A straightforward computation gives $\lim _{x_{d} \rightarrow x_{c}}\left(x_{c}-x_{d}\right) \Psi_{w^{\prime} c d w^{\prime \prime}}=\Psi_{w^{\prime} c w^{\prime \prime}}$. The result follows.

Theorem 3.1 and Proposition 2.13 show that the knowledge of the fraction $\Psi_{P}$ for each bipartite poset is sufficient to compute any $\Psi_{P}$ by applying a sequence of residues. In fact, we can construct other algorithms like the decontraction to obtain bipartite posets with such properties. However, we will show that the decontraction implies a stronger result (Proposition 3.10, Section 3.4). 


\subsection{Limits and suppression of extremal elements}

The deletion of a vertex admits also an interpretation in terms of rational functions.

Theorem 3.2 Let $c$ be an element of a poset $P$,

$$
\lim _{x_{c} \rightarrow+\infty}\left(x_{c} \cdot \Psi_{P}\right)= \begin{cases}-\Psi_{P \backslash c} & \text { if } c \text { is maximal }, \\ \Psi_{P \backslash c} & \text { if } c \text { is minimal }, \\ 0 & \text { otherwise. }\end{cases}
$$

Proof: We have

$$
\lim _{x_{c} \rightarrow+\infty}\left(x_{c} \cdot \Psi_{P}\right)=\lim _{x_{c} \rightarrow+\infty} \sum_{w \in \mathcal{L}(P)}\left(x_{c} \cdot \Psi_{w}\right) .
$$

We split the sum into two parts, the first one being the sum over all the words beginning or finishing by $c$

$$
\lim _{x_{c} \rightarrow+\infty}\left(x_{c} \cdot \Psi_{P}\right)=\lim _{x_{c} \rightarrow+\infty}\left(\sum_{\substack{w \in \mathcal{L}(P) \\ w=c w^{\prime} \text { or } w=w^{\prime} c}}\left(x_{c} \cdot \Psi_{w}\right)+\sum_{\substack{w \in \mathcal{L}(P), w_{w} w^{\prime} c w^{\prime \prime} \\ \text { and } w^{\prime}, w^{\prime \prime} \text { are non-trivial }}}\left(x_{c} \cdot \Psi_{w}\right)\right) .
$$

If $c$ is the first or the last letter in $w$, then the degree of $x_{c}$ in $\psi_{w}$ is -1 and $\lim _{x_{c} \rightarrow+\infty}\left(x_{c} \psi_{w}\right)= \pm \psi_{w^{\prime}}$. If $c$ is not in the first and last position in $w$, then the degree of $x_{c}$ in $\psi_{w}$ is -2 and $\lim _{x_{c} \rightarrow+\infty}\left(x_{c} \psi_{w}\right)=0$. We deduce that limit and sums in Equation (5) can be interchanged to obtain

$$
\lim _{x_{c} \rightarrow+\infty}\left(x_{c} \cdot \Psi_{P}\right)=\sum_{\substack{w \in \mathcal{L}(P) \\ w=c w^{\prime} \text { or } w=w^{\prime} c}} \lim _{x_{c} \rightarrow+\infty}\left(x_{c} \cdot \Psi_{w}\right) .
$$

We get

$$
\lim _{x_{c} \rightarrow+\infty}\left(x_{c} \cdot \Psi_{P}\right)=\sum_{\substack{w \in \mathcal{L}(P) \\ w=c w^{\prime} \text { or } w=w^{\prime} c}} \Psi_{w^{\prime}}
$$

By Proposition 2.15, we have

$$
\lim _{x_{c} \rightarrow+\infty}\left(x_{c} \cdot \Psi_{P}\right)= \pm \Psi_{P \backslash\{c\}}
$$

\subsection{Connectivity and vanishing conditions}

In this subsection, we investigate the interpretation of connectivity in terms of rational functions. In particular, Corollary 3.6 shows that $\Psi_{P}=0$ if and only if $P$ is disconnected.

Definition 3.3 (Greene (3)) A poset $P$ is planar if its Hasse diagram may be ordered-embedded in $\mathbb{R} \times \mathbb{R}$ without edge crossings, even when extra maximal and minimal elements are added.

Example 3.4 Following the definition of Greene (3), Figures 9 and 10 are examples of non-planar and planar posets, respectively. 


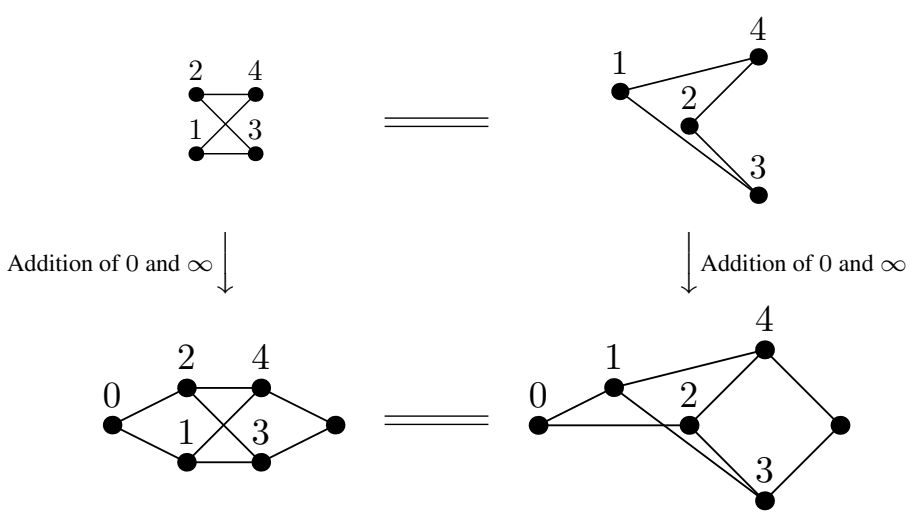

Fig. 9: The poset $\mathbb{P}_{2143}$ is not planar.



Fig. 10: The poset $\mathbb{P}_{1324}$ is planar.

Theorem 3.5 (Greene (3)) Let $P$ be a planar poset, then

$$
\Psi_{P}=\left\{\begin{array}{cl}
0 & \text { if } P \text { is not connected } \\
\prod_{\substack{a, b \in P \\
a<b}}\left(x_{a}-x_{b}\right)^{\mu_{P}(a, b)} & \text { if } P \text { is connected }
\end{array}\right.
$$

where $\mu_{P}$ denotes the Möbius function of $P$.

As a consequence, one has:

Corollary 3.6 Let $P$ be a poset, the Hasse diagram of $P$ is connected if and only if $\Psi_{P} \neq 0$.

Proof: Suppose first that $P$ is connected and $\Psi_{P}=0$. Since contractions preserve the connectivity of a poset, we contract successively the edges of $P$ until we obtain a new poset with only two elements $c \leq d$. Using Theorem 3.1, we get $\Psi_{c-d}$ from $\Psi_{P}$ by applying a succession of residues. It follows that $\Psi_{c-d}=0$. This is in contradiction with the direct computation $\Psi_{c-d}=\frac{1}{x_{c}-x_{d}}$. Hence, $\Psi_{P} \neq 0$.

Conversely, we consider the case of a disconnected poset $P$. Let $C_{1}$ and $C_{2}$ be two disconnect components of $P$. Applying Lemma 2.5, one gets:

$$
\mathcal{L}(P)=\bigsqcup_{w_{1} \in \mathcal{L}\left(C_{1}\right), w_{2} \in \mathcal{L}\left(C_{2}\right)} \mathcal{L}\left(\left(P_{w_{1}}\right)_{w_{2}}\right) .
$$


The disjoint union interpreted as a sum, when stated in terms of rational function, gives

$$
\Psi_{P}=\sum_{w_{1} \in \mathcal{L}\left(C_{1}\right), w_{2} \in \mathcal{L}\left(C_{2}\right)} \Psi_{\left(P_{w_{1}}\right)_{w_{2}}} .
$$

Since each poset $\left(P_{w_{1}}\right)_{w_{2}}$ is planar and disconnected, Greene's theorem (Theorem 3.5 gives $\Psi_{P}=0$.

\subsection{Reduced fractions and Hasse diagrams}

In this subsection, we present two main results on $\Psi_{P}$. We characterise the denominator of the reduced fraction $\Psi_{P}$ with the help of the Hasse diagram of $P$, and we give an interpretation of the degree of the numerator of $\Psi_{P}$ in terms of cycles.

We call cycles (resp. cyclomatic number) of a poset $P$, the cycles (resp. cyclomatic number) of the Hasse diagram of $P$. We recall that the cyclomatic number of a graph $G$ is the minimal number of edges we have to remove from $G$ to obtain a graph without cycle (see e.g. (8) or (9)).

We denote by $\operatorname{Den}\left(\Psi_{P}\right)$ the denominator of the reduced fraction $\Psi_{P}$ and by $\operatorname{Num}\left(\Psi_{P}\right)$ its numerator.

Corollary 3.7 Let $P$ be a connected poset, then:

$$
\operatorname{Den}\left(\Psi_{P}\right)=\prod_{c \prec d}\left(x_{c}-x_{d}\right) .
$$

Proof: Theorem 3.1 implies that $\prod_{c \prec d}\left(x_{c}-x_{d}\right)$ is a factor of $\operatorname{Den}\left(\Psi_{P}\right)$. Since contractions preserve the connectivity (Corollary 3.6, we deduce that $\operatorname{Den}\left(\Psi_{P}\right)$ is exactly $\prod_{c \prec d}\left(x_{c}-x_{d}\right)$.

This result shows that the Hasse diagram is a relevant notion in our context. Moreover, the following corollary confirms the special status of the Hasse diagram and in particular of its cycles.

Corollary 3.8 Let $P$ be a connected poset, the degree of $N u m\left(\Psi_{P}\right)$ is equal to the cyclomatic number of the Hasse diagram of $P$.

Proof: Let $P$ be a connected poset with $n$ elements. Let $m$ be the number of edges in $H(P)$ and $o$ its cyclomatic number. By Corollary 3.7, we deduce that the degree of the numerator of the reduced fraction is at most equal to $m-n+1$ that is, from the Euler formula, (see e.g. (8) or (9)), the cyclomatic number of $H(P)$. The polynomial $N u m(P)$ being homogeneous, it equals either zero or its degree is $o$. Since $P$ is connected, Corollary 3.6 finishes the proof.

Example 3.9 The cyclomatic number of the Hasse diagram of $P_{132546}$ (see Figure 11) is 3. So the degree of its numerator equals 3.

$$
\begin{aligned}
& \operatorname{Num}\left(\Psi_{\mathbb{P}_{132546}}\right)=x_{1} \cdot x_{2} \cdot x_{3}-x_{1} \cdot x_{2} \cdot x_{6}-x_{2} \cdot x_{3} \cdot x_{4}-x_{1} \cdot x_{3} \cdot x_{6}-x_{1} \cdot x_{4} \cdot x_{5}-x_{2} \cdot x_{3} \cdot x_{5}+ \\
& x_{1} \cdot x_{4} \cdot x_{6}+x_{2} \cdot x_{3} \cdot x_{6}+x_{2} \cdot x_{4} \cdot x_{5}+x_{1} \cdot x_{5} \cdot x_{6}+x_{3} \cdot x_{4} \cdot x_{5}-x_{4} \cdot x_{5} \cdot x_{6} \\
& \operatorname{Den}\left(\Psi_{\mathbb{P}_{132546}}\right)=\left(x_{1}-x_{3}\right) \cdot\left(x_{1}-x_{2}\right) \cdot\left(x_{5}-x_{6}\right) \cdot\left(x_{4}-x_{6}\right) \cdot\left(x_{3}-x_{5}\right) \cdot\left(x_{2}-x_{5}\right) \cdot\left(x_{3}-x_{4}\right) \cdot\left(x_{2}-x_{4}\right)
\end{aligned}
$$




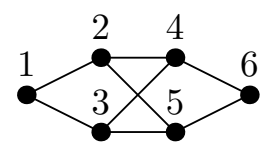

Fig. 11: Numerator and denominator of the reduced fraction $\Psi_{\mathbb{P}_{132546}}$.

From Theorem 3.1 and Theorem 2.13, we know that any rational function of a poset $P$ can be calculated from the bipartite poset $\bar{P}$ by applying a sequence of residues. In fact, due to the construction of $\bar{P}$, the following proposition gives a stronger result.

Proposition 3.10 Let $P$ be a poset and $\bar{P}$ its decontraction, then any sequence of limits setting $x_{a}$ to $x_{\bar{a}}$ for all elements $a \in \operatorname{In}(P)$ send $\operatorname{Num}\left(\Psi_{\bar{P}}\right)$ to $\operatorname{Num}\left(\Psi_{P}\right)$, i.e.,

$$
\operatorname{Num}\left(\Psi_{P}\right)=\lim _{\substack{x_{\bar{a} \rightarrow x} \\ a \in \operatorname{In}(P)}} \operatorname{Num}\left(\Psi_{\bar{P}}\right)
$$

Proof: By construction of $\bar{P}$ (see Theorem 2.13, we can apply to $\bar{P}$ any sequence of contractions of edges $(a, \bar{a})$ where $a$ is an element of $\operatorname{In}(P)$. The poset obtained is exactly $P$ and by Theorem 3.1, we have:

$$
\Psi_{P}=\lim _{\substack{x_{\bar{a}} \rightarrow x_{a} \\ a \in \operatorname{In}(P)}} \prod_{a \in \operatorname{In}(P)}\left(x_{a}-x_{\bar{a}}\right) \cdot \Psi_{\bar{P}}
$$

From Corollary 3.7, we have

$$
\Psi_{P}=\lim _{\substack{x_{\bar{a}} \rightarrow x_{a} \\ a \in \operatorname{In}(P)}} \frac{\operatorname{Num}\left(\Psi_{\bar{P}}\right) \cdot \prod_{a \in \operatorname{In}(P)}\left(x_{a}-x_{\bar{a}}\right)}{\prod_{(a, b) \in H(\bar{P})}\left(x_{a}-x_{b}\right)} .
$$

By construction of $\bar{P}$,

$$
\left.(H(\bar{P}) \backslash\{(a, \bar{a}) \mid a \in \operatorname{In}(P)\})\right|_{\bar{a}=a}=H\left(\left.\bar{P}\right|_{\bar{a}=a}\right)=H(P) .
$$

Consequently,

$$
\frac{\prod_{a \in \operatorname{In}(P)}\left(x_{a}-x_{\bar{a}}\right) \cdot \operatorname{Num}\left(\Psi_{\bar{P}}\right)}{\prod_{(a, b) \in H(\bar{P})}\left(x_{a}-x_{b}\right)}=\frac{\operatorname{Num}\left(\Psi_{\bar{P}}\right)}{\prod_{(a, b) \in H(\bar{P}) \backslash\{(a, \bar{b}) \mid a \in \operatorname{In}(P)\}}\left(x_{a}-x_{b}\right)} .
$$

Substituting Equations (7) and (8) in Equation (6), we obtain

$$
\Psi_{P}=\frac{\lim _{\substack{x \rightarrow x_{a} \\ a \in \operatorname{In}(P)}} \operatorname{Num}\left(\Psi_{\bar{P}}\right)}{\prod_{(a, b) \in H(P)}\left(x_{a}-x_{b}\right)} .
$$


Moreover, using Corollary 3.7, we conclude

$$
\operatorname{Num}\left(\Psi_{P}\right)=\lim _{\substack{x_{a} \rightarrow x_{a} \\ a \in \operatorname{In}(P)}} \operatorname{Num}\left(\Psi_{\bar{P}}\right)
$$

Example 3.11 The rational functions $\Psi_{\overline{\mathbb{P}_{21435}}}$ and $\Psi_{\mathbb{P}_{21435}}$ (see Figure 12 ) have the following numerators and denominators:

$\operatorname{Num}\left(\Psi_{\overline{\mathbb{P}_{21435}}}\right)=x_{5} \cdot x_{\overline{3}}-x_{1} \cdot x_{\overline{4}}-x_{2} \cdot x_{\overline{3}}-x_{2} \cdot x_{\overline{4}}-x_{3} \cdot x_{\overline{3}}-x_{3} \cdot x_{\overline{4}}-x_{4} \cdot x_{\overline{3}}-x_{4} \cdot x_{\overline{4}}-x_{1} \cdot x_{\overline{3}}+$ $x_{5} \cdot x_{\overline{4}}+x_{\overline{3}}^{2}+x_{\overline{4}}^{2}+x_{\overline{3}} \cdot x_{\overline{4}}+x_{1} \cdot x_{2}+x_{1} \cdot x_{3}+x_{1} \cdot x_{4}+x_{2} \cdot x_{3}-x_{1} \cdot x_{5}+x_{2} \cdot x_{4}-x_{2} \cdot x_{5}$

$$
\operatorname{Num}\left(\Psi_{\mathbb{P}_{21435}}\right)=x_{1} \cdot x_{2}+x_{3} \cdot x_{5}+x_{4} \cdot x_{5}-x_{1} \cdot x_{5}-x_{2} \cdot x_{5}-x_{3} \cdot x_{4}
$$

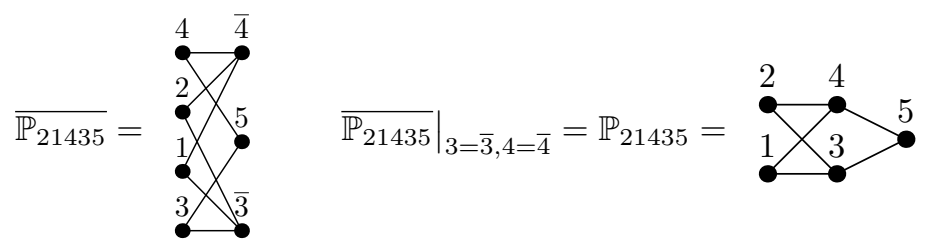

Fig. 12: We obtain the fraction $\Psi_{\mathbb{P}_{21435}}$ from $\Psi_{\overline{\mathbb{P}_{21435}}}$.

\subsection{Collapses and factorisations}

In general, when $a$ is an extremal element of $P$, the knowledge of $\Psi_{P \backslash a}$ is not sufficient to compute $\Psi_{P}$. However, collapse is a special suppression which allows us to calculate $\Psi_{P}$ from $\Psi_{P \backslash a}$.

Proposition 3.12 Let $a$ be an element of a connected poset $P$ such that a is a free vertex in the Hasse diagram of $P$. Let $b$ be the unique vertex such that either $b \preceq a$ or $a \preceq b$. Then we have

$$
\Psi_{P}= \begin{cases}\Psi_{P \backslash\{a\}} \cdot \frac{1}{x_{a}-x_{b}} & \text { if a is minimal, } \\ \Psi_{P \backslash\{a\}} \cdot \frac{1}{x_{b}-x_{a}} & \text { if a is maximal. }\end{cases}
$$

Proof: Let $a$ be a free vertex in the Hasse diagram of $P$. We write the numerator of $\Psi_{P}$ as a polynomial in $x_{a}$ with coefficients in $\mathbb{C}\left[\mathbb{X} \backslash x_{a}\right]$ :

$$
\operatorname{Num}\left(\Psi_{P}\right)=\sum_{i} C_{i} x_{a}^{i}
$$

Since $a$ is a free vertex, $a$ is either maximal or minimal in $P$. Theorem 3.2 shows that

$$
\lim _{x_{a} \rightarrow+\infty}\left(x_{a} \cdot \Psi_{P}\right)= \begin{cases}-\Psi_{P \backslash a} & \text { if } a \text { is maximal, } \\ \Psi_{P \backslash a} & \text { if } a \text { is minimal, }\end{cases}
$$

which implies that $C_{0} \neq 0$ and for all $i \geq 1, C_{i}=0$. Hence, $\operatorname{Num}\left(\Psi_{P}\right)=C_{0}=\operatorname{Num}\left(\Psi_{P \backslash\{a\}}\right)$.

As a straightforward consequence, we obtain the following result. 
Corollary 3.13 $\operatorname{Num}\left(\Psi_{P}\right)=\operatorname{Num}\left(\Psi_{\text {Coll }(P)}\right)$, where, as before, Coll $(P)$ denotes the collapse of $P$.

\section{Examples}

In general we do not know how to compute $\Psi_{P}$. But, for some special cases, we can give a closed formula.

\subsection{Acyclic posets}

Proposition 4.1 The Hasse diagram $H(P)$ has no cycle if and only if $N u m(P)=1$.

Proof: This result is a direct consequence of Greene's theorem (Theorem 3.5 ) and Corollary 3.8

A permutation $\sigma$ avoids the patterns 1324 if there exist no integers $1 \leq i_{1}<i_{2}<i_{3}<i_{4} \leq n$ such that $\sigma_{i_{1}}<\sigma_{i_{3}}<\sigma_{i_{2}}<\sigma_{i_{4}}$. A permutation $\sigma$ contains the pattern $\overline{2143}$ if for some indices $1 \leq i_{1}<i_{2}<i_{3}<i_{4} \leq n$ we have $\sigma_{i_{2}} \leq \sigma_{i_{1}} \leq \sigma_{i_{4}} \leq \sigma_{i_{3}}$ with the further restriction that there is no $i_{1} \leq j \leq i_{4}$ such that $\sigma_{i_{1}} \leq \sigma_{j} \leq \sigma_{i_{4}}$.

Butler and Bousquet-Mélou have shown in (1) that the Hasse diagram of a poset associated to a permutation avoiding 1324 and $\overline{2143}$ has no cycle. As a consequence we have the following corollary.

Corollary 4.2 We have $\operatorname{Num}\left(\Psi_{\mathbb{P}_{\sigma}}\right)=1$ if and only if $\sigma$ avoids the patterns 1324 and $\overline{2143}$.

\subsection{1-cycle posets}

Proposition 4.3 Let $P$ be a connected 1-cycle poset, then

$$
\operatorname{Num}(P)=\sum_{a \in \min (\operatorname{Coll}(P))} x_{a}-\sum_{a \in \max (\operatorname{Coll}(P))} x_{a} .
$$

Proof: Consider the poset $P^{\prime}=\overline{\operatorname{Coll}(P)}$ obtained by the construction given in Theorem 2.13 applied to $\operatorname{Coll}(P)$.

Since $P^{\prime}$ is bipartite with only 1 cycle, by Corollary 3.8 we obtain $N u m\left(\Psi_{P^{\prime}}\right)=\sum_{a} \beta_{a} x_{a}$, where $\beta_{a} \in \mathbb{Z}$. Let $a$ be a minimal element in $P^{\prime}$. Since $P^{\prime} \backslash\{a\}$ is acyclic and connected, $\operatorname{Num}\left(\Psi_{P^{\prime} \backslash\{a\}}\right)=1$. Theorem 3.2 implies that $\beta_{a}=1$ if $a$ is maximal and $\beta_{y}=-1$ if $a$ is minimal. So, we have:

$$
\operatorname{Num}\left(\Psi_{P^{\prime}}\right)=\sum_{a \in \min \left(P^{\prime}\right)} x_{a}-\sum_{a \in \max \left(P^{\prime}\right)} x_{a} .
$$

By Proposition 3.10, we have:

$$
\operatorname{Num}\left(\Psi_{P}\right)=\lim _{\substack{x_{a} \rightarrow x_{a} \\ a \in \operatorname{In}\left(P^{\prime}\right)}} \operatorname{Num}\left(\Psi_{P^{\prime}}\right) .
$$

Hence,

$$
\begin{aligned}
\operatorname{Num}\left(\Psi_{P}\right) & =\sum_{a \in \min (\operatorname{Call}(P))} x_{a}-\sum_{a \in \max (\operatorname{Call}(P))} x_{a}+\sum_{\substack{a \in \operatorname{In}(\operatorname{Call}(P)) \\
\sum_{a \in \operatorname{In}(\operatorname{Call}(P))}}} x_{a}-\sum_{a \in \max (P))}\left(x_{a}-x_{\bar{a}}\right) \\
& =x_{a} .
\end{aligned}
$$


Example 4.4 The numerator of the 1-cycle poset P in Figure 13 is

$$
\operatorname{Num}\left(\Psi_{P}\right)=x_{1}+x_{2}-x_{4}-x_{7} .
$$

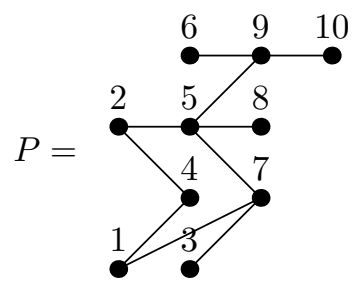

Fig. 13: 1-cycle poset.

\section{$4.3 \lambda$-complete posets and Schubert polynomials}

We have seen in Subsection 3.1 that the bipartite posets are fundamental for the description of the functions $\Psi_{P}$. In this section, we compute $\Psi_{\mathcal{P}}$ for special cases of bipartite posets.

Let $\lambda$ be a partition (a weakly decreasing sequence of non-negative integers). We call a bipartite poset with elements $\left\{a_{1}, \ldots, a_{l(\lambda)}, b_{1}, \ldots, b_{\lambda_{1}}\right\}$ and relation $\leq_{\lambda}$ a $\lambda$-complete poset, if the relation $\leq_{\lambda}$ is defined by $a_{i} \leq_{\lambda} b_{j}$ if and only if $j \leq \lambda_{i}$. We will write $P^{\lambda}$ for this poset.

For example, Figure 14 shows a 5433-complete poset.

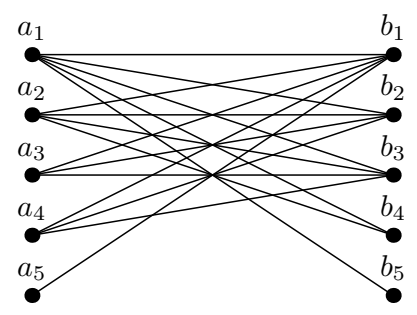

Fig. 14: The 54331-complete poset.

Let $f\left(a_{1}, \ldots, a_{n}\right)$ be a polynomial in the alphabet $A=\left\{a_{1}, \ldots, a_{n}\right\}$. The divided differences $\partial_{i}$ (see (4)) is the operator defined by

$$
f \cdot \partial_{i}=\frac{f-f^{s_{i}}}{x_{a_{i}}-x_{a_{i+1}}},
$$

where $f^{s_{i}}$ is the function $f\left(a_{1}, \ldots, a_{i+1}, a_{i}, \ldots, a_{n}\right)$.

We recall that the definition of Schubert polynomials as it can be found in (4).

Schubert polynomials $\mathbb{Y}_{v}(A, B)$ are functions in the alphabets $A=\left\{a_{1}, a_{2}, \ldots\right\}$ and $B=\left\{b_{1}, b_{2}, \ldots\right\}$ indexed by vectors $v \in \mathbb{N}^{\infty}$ having a finite number of non-zero components and defined recursively by: 
1. if $v$ is weakly decreasing then,

$$
\mathbb{Y}_{v}(\mathbb{A}, \mathbb{B}):=\prod_{i=1}^{l(v)} \prod_{j=1}^{v_{i}}\left(x_{a_{i}}-x_{b_{j}}\right)
$$

2. if there exists $i \in \mathbb{N}$ such that $v_{i}<v_{i+1}$ then,

$$
\mathbb{Y}_{v} \cdot \partial_{i}:=\mathbb{Y}_{v \cdot \partial_{i}}
$$

where $v \cdot \partial_{i}=\left(v_{1}, \ldots, v_{i-1}, v_{i+1}, v_{i}-1, v_{i+2}, \ldots\right)$.

Proposition 4.5 Let $\lambda=\lambda_{1} \ldots \lambda_{n}$ be a partition with $\lambda_{n}>0$. For any $\lambda$-complete poset $\mathcal{P}^{\lambda}$, the numerator of $\Psi_{\mathcal{P}^{\lambda}}$ is the Schubert polynomial

$$
\operatorname{Num}\left(\Psi_{\mathcal{P}^{\lambda}}\right)=\mathbb{Y}_{0, \lambda_{2}-1, \ldots, \lambda_{n}-1}
$$

Proof: Let $A$ and $B$ be two alphabets, and let $X$ be a subalphabet of $A$. Let $\lambda$ be a partition and $k$ an integer with $\lambda_{k}>1$ and $\lambda_{k+1} \leq 1$. Let $\left(A^{i}\right)_{i \in[1, k-1]}$ be the family of alphabets defined by $A^{i}=$ $\left\{a_{i}, \ldots, a_{k}\right\}$. Let $\left(\beta^{i}\right)_{i \in[1, k-1]}$ be the family of partitions defined by $\beta^{i}=\left[\lambda_{i+1}, \lambda_{i+1}, \ldots, \lambda_{k}\right]$. We will

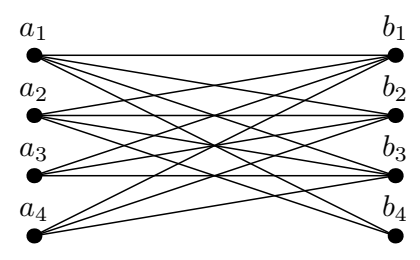

Fig. 15: Poset $\mathcal{P}^{\beta^{1}}$ for $\lambda=54331$ (which implies $\beta^{1}=4433$ ).

denote by $\mathcal{P}^{\lambda}(X)$ the $\lambda$-complete poset on $X$ and $B$ :

$$
\mathcal{P}^{\lambda}(X):=\mathcal{P}^{\lambda}(X, B)
$$

We will prove the following recursive formula:

$$
\operatorname{Num}\left(\Psi_{\mathcal{P}^{\beta^{i}}\left(A^{i}\right)}\right)=\left(\mathbb{Y}_{\lambda_{i+1}}\left(a_{i}\right) \cdot \operatorname{Num}\left(\Psi_{\mathcal{P}^{\beta^{i+1}}}\left(A^{i+1}\right)\right)\right) \partial_{i}
$$

We denote by $P^{\prime}$ the expression $\mathcal{P}^{\beta^{i}}\left(A^{i}\right)$. By Lemma 2.5 , we sort $a_{i}$ and $a_{i+1}$ to obtain

$$
\Psi_{P^{\prime}}=\Psi_{P_{a_{i} a_{i+1}}^{\prime}}+\Psi_{P_{a_{i+1} a_{i}}^{\prime}} .
$$

The Hasse diagram of $P_{a_{i} a_{i+1}}^{\prime}$ is obtained from the Hasse diagram of $P^{\prime}$ by removing all the edges having $a_{i}$ as vertex and adding the edge $\left(a_{i}, a_{i+1}\right)$. In the same way, the Hasse diagram of $P_{a_{i+1}, a_{i}}^{\prime}$ can be obtained by applying the transposition $s_{i}$ on $P_{a_{i}, a_{i+1}}^{\prime}$. 


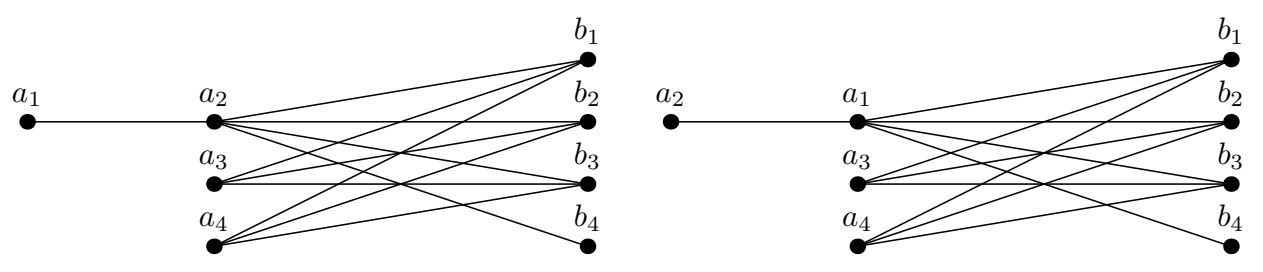

Fig. 16: $a_{1}$ and $a_{2}$ are sorted in $\beta^{1}$-complete poset, where $\lambda=54331$ (which implies $\beta^{1}=4433$ ).

The vertex $a_{i}$ (respectively $a_{i+1}$ ) is free in $P_{a_{i} a_{i+1}}^{\prime}$ (respectively $P_{a_{i+1} a_{i}}^{\prime}$ ) and can be collapsed. By Proposition 3.12 and Corollary 3.7 we obtain

$$
\left.\Psi_{P^{\prime}}=\frac{1}{x_{a_{i}}-x_{a_{i+1}}} \cdot\left(\Psi_{\mathcal{P}_{2}^{\left[\beta_{2}^{i} \ldots \beta_{k}^{i}\right]}\left(A_{i} \backslash\left\{a_{i}\right\}\right)}-\Psi_{\mathcal{P}^{\left[\beta_{2}^{i} \ldots \beta_{k}^{i}\right]}\left(A_{i} \backslash\left\{a_{i+1}\right\}\right)}\right)\right) .
$$

Observing

$$
\mathbb{Y}_{\lambda_{i+1}}(x)=\prod_{j=1}^{\lambda_{i+1}} x-x_{b_{j}},
$$

and using Corollary 3.7 in Equation 10 , we obtain:

$$
\Psi_{P^{\prime}}=\frac{1}{x_{a_{i}}-x_{a_{i+1}}} \cdot \frac{\mathbb{Y}_{\lambda_{i+1}}\left(a_{i}\right) \cdot \operatorname{Num}\left(\Psi_{\mathcal{P}^{\left[\beta_{2}^{i} . \beta_{k}^{i}\right]}\left(A \backslash\left\{a_{i}\right\}\right)}\right)-\mathbb{Y}_{\lambda_{i+1}}\left(a_{i+1}\right) \cdot \operatorname{Num}\left(\Psi_{\mathcal{P}_{2}^{\left[\beta_{2}^{i} . \beta_{k}^{i}\right]}\left(A \backslash\left\{a_{i+1}\right\}\right.}\right)}{\prod_{c \preceq d}\left(x_{c}-x_{d}\right)} .
$$

Hence,

$$
\operatorname{Num}\left(\Psi_{\mathcal{P}^{\beta^{i}}\left(A^{i}\right)}\right)=\left(\mathbb{Y}_{\lambda_{i+1}}\left(a_{i}\right) \cdot \operatorname{Num}\left(\Psi_{\mathcal{P}^{\left[\beta_{2}^{i} \ldots \beta_{k}^{i}\right]}\left(A^{i+1}\right)}\right)\right) \partial_{i} .
$$

If we collapse the poset $\mathcal{P}^{\left[\beta_{2}^{i} \ldots \beta_{k}^{i}\right]}\left(A^{i+1}\right)$, we obtain the poset $\mathcal{P}^{\beta^{i+1}}\left(A^{i+1}\right)$ and we can conclude that

$$
\operatorname{Num}\left(\Psi_{\mathcal{P}^{\beta^{i}}\left(A^{i}\right)}\right)=\left(\mathbb{Y}_{\lambda_{i+1}}\left(a_{i}\right) \cdot \operatorname{Num}\left(\Psi_{\mathcal{P}^{\beta^{i+1}}\left(A^{i+1}\right)}\right)\right) \partial_{i}
$$

From Equation (12) we obtain

$$
\operatorname{Num}\left(\Psi_{\mathcal{P}^{\beta^{1}}}\right)=\left(\mathbb{Y}_{\lambda_{2}}\left(a_{1}\right) \cdots \mathbb{Y}_{\lambda_{k-1}}\left(a_{k-2}\right) \operatorname{Num}\left(\Psi_{\mathcal{P}^{\lambda_{k} \lambda_{k}}\left(\left\{a_{k-1}, a_{k}\right\}\right)}\right)\right) \partial_{k-2} \cdots \partial_{1} .
$$

Using Equation (11), we deduce that

$$
\operatorname{Num}\left(\Psi_{\mathcal{P}^{\lambda_{k} \lambda_{k}}\left(\left\{a_{k-1}, a_{k}\right\}\right)}\right)=\mathbb{Y}_{\lambda_{k}}\left(a_{k-1}\right) \operatorname{Num}\left(\Psi_{\mathcal{P}^{\lambda_{k}}\left(\left\{a_{k}\right\}\right)}\right) .
$$

Since $\mathcal{P}^{\lambda_{k}}\left(\left\{a_{k}\right\}\right)$ has no cycle, $\operatorname{Num}\left(\Psi_{\mathcal{P}^{\lambda_{k}}\left(\left\{a_{k}\right\}\right)}\right)=1$ and

$$
\operatorname{Num}\left(\Psi_{\mathcal{P}^{\beta^{1}}}\right)=\left(\mathbb{Y}_{\lambda_{2}}\left(a_{1}\right) \cdots \mathbb{Y}_{\lambda_{k}}\left(a_{k-1}\right)\right) \partial_{k-1} \cdots \partial_{1} .
$$

Finally, we obtain the result

$$
\operatorname{Num}\left(\Psi_{\mathcal{P}^{\beta^{1}}}\right)=\mathbb{Y}_{0, \lambda_{2}-1, \ldots, \lambda_{k}-1} .
$$


It is easy to see that $\mathcal{P}^{\lambda}=\operatorname{Coll}\left(\mathcal{P}^{\beta^{1}}\right)$. We deduce that,

$$
\operatorname{Num}\left(\Psi_{\mathcal{P}^{\lambda}}\right)=\mathbb{Y}_{0, \lambda_{2}-1, \ldots, \lambda_{k}-1}=\mathbb{Y}_{0, \lambda_{2}-1, \ldots, \lambda_{n}-1} .
$$

\section{Acknowledgements}

The author is grateful to A. Lascoux for his suggestion to work on rational functions and his introduction to Schur polynomials. The author thanks J.G. Luque for useful discussions and revisions of this paper. The author thanks T. Gomez-Diaz for critical reading of this article.

\section{References}

[1] S. Butler and M. Bousquet-Mélou, Forest-like permutations, Ann. Comb. 11 (2007), 335-354.

[2] A. Cortez, Singularités génériques et quasi-résolutions des variétés de Schubert pour le groupe linéaire, Adv. Math. 178 (2003), 396-445.

[3] C. Greene, A rational function identity related to the Murnaghan-Nakayama formula for the characters of $\mathcal{S}_{n}$, J. Alg. Comb. 13 (1992), 235-255.

[4] A. Lascoux, Symmetric Functions and Combinatorial Operators on Polynomials, Conference Board of the Mathematical Sciences Series, Amer. Math. Soc. 99 (2003).

[5] A.M. Fu and A. Lascoux, q-Identities from Lagrange and Newton interpolation, Adv. App. Math. 31 (2003), 527-531.

[6] A. Woo and A. Yong, When is a Schubert variety Gorenstein?, Adv. Math. 207 (2006), 205-220.

[7] I. G. Macdonald, Symmetric Functions and Hall Polynomials, Oxford University Press, 2nd edition (1998).

[8] R. Diestel, Graph Theory, Graduate Texts in Mathematics 173 (2005), Springer-Verlag Heidelberg.

[9] P. J. Giblin, Graphs, Surface and Homology - An Introduction to Algebraic Topology (1981), London and New York Chapman and Hall. 